\title{
An expeditious one-pot solvent-free synthesis of benzimidazole derivatives
}

\author{
Harjyoti Thakuria and Gopal Das* \\ Department of Chemistry, Indian Institute of Technology Guwahati, Assam 781039, India \\ E-mail:gdas@iitgernet.in
}

\begin{abstract}
A one-pot efficient synthesis of biologically active benzimidazole derivatives has been achieved under solvent-free green conditions, with unsurpassed atom economy, by a simple grinding method followed by heating.
\end{abstract}

Keywords: Green synthesis, grinding, atom economy, o-phenylenediamines, benzimidazole, 2mercaptobenzimidazole

\section{Introduction}

Benzimidazoles are very useful intermediates/subunits for the development of molecules of pharmaceutical or biological interest. ${ }^{1}$ Benzimidazole and its derivatives are an important class of bioactive molecules in the field of drugs and pharmaceuticals. ${ }^{2}$ Benzimidazole derivatives have found applications in diverse therapeutic areas including anti-ulcers, anti-hypertensives, anti-virals, anti-fungals, anti-cancers, and anti-histaminics. ${ }^{2}$ Moreover, these fused heterocycles have been studied as new non-nucleoside topoisomerase-I poisons, human immunodeficiency virus-1 reverse transcriptase inhibitors, and/or potent DNA gyrase inhibitors. ${ }^{3}$ They can act as ligands to transition metals for modeling biological systems. ${ }^{4}$ In addition, benzimidazoles are very important intermediates in organic reactions. ${ }^{5}$

Due to their wide range of pharmacological activity, industrial- and synthetic applications, a number of methods have been reported for the synthesis of benzimidazole and its derivatives. These methods include the coupling of $o$-phenylenediamines and carboxylic acids or various derivatives. ${ }^{6}$ Various catalyzed syntheses of benzimidazole derivatives are known, by condensation of $o$-phenylenediamine with aldehydes. ${ }^{7}$ Condensation of $o$-phenylenediamine with ortho-esters in the presence of various Lewis acid catalyst is also known viz. $\mathrm{ZrCl}_{4}, \mathrm{SnCl}_{4} .5 \mathrm{H}_{2} \mathrm{O}$, $\mathrm{TiCl}_{4}, \mathrm{BF}_{3} . \mathrm{Et}_{2} \mathrm{O}, \mathrm{ZrOCl}_{2} .8 \mathrm{H}_{2} \mathrm{O}$ and $\mathrm{HfCl}_{4}{ }^{8}$ Phthalic acid attached to polyethylene glycol ether and polymer supported 4-fluoro-3-nitrobenzoic acid in solid phase have been used as precursor for 2-substituted benzimidazoles, benzoxazoles and benzothiazoles. ${ }^{9}$ However, all of these 
methods have problems, including drastic reaction conditions, low yields and severe sidereactions. Catalytic synthetic methods required tedious work up procedures such as filtration, purification and drying for reuse of the catalyst. Moreover, more than one step is involved in the synthesis of these compounds in some procedures. Therefore, the pursuance of more convenient and practical synthetic methods for these compounds still remains an active research area.

2-Mercaptobenzimidazole derivatives are known to possess varied biological activities. ${ }^{10}$ Thus, syntheses of these series of compounds are highly desirable. Condensations of $o-$ phenylenediamine with carbon disulfide in the presence of alkali $\mathrm{KOH}$, with thiourea using polyphosphoric acid (PPA) as a catalyst, with sulfur (S8) using $\mathrm{Bu}_{4} \mathrm{NOH}$ as a catalyst are known. ${ }^{11}$

\section{Results and Discussion}

In continuation of our interest to explore the utility of simple one-pot, solvent-free, solid phase grinding methods, ${ }^{12}$ we decided to investigate the efficient synthesis of biologically active benzimidazole derivatives. Herein, we report the efficient one-pot solvent-free green synthesis of a series of benzimidazole derivatives in good yield at $140^{\circ} \mathrm{C}$. We also report the one-pot efficient synthesis of other biologically potent compounds, 2-mercaptobenzimidazole derivatives under solvent-free conditions. In this methodology, both components were thoroughly ground with a pestle in a mortar at room temperature in an open atmosphere until the mixture turned into a melt. For liquid starting materials, they were mixed thoroughly for a given period of time instead of grinding.

At first, we synthesized benzimidazole derivatives by coupling o-phenylenediamine either with organic acid (Table 1) or aldehyde in solvent-free neat conditions (Table 2). In order to ascertain the optimum temperature, several reactions were carried out on o-phenylenediamine and acetic acid as the model by varying the reaction temperature and finally the best result was obtained at $140^{\circ} \mathrm{C}$. Having established the reaction temperature, various organic acids and aldehydes were subjected to coupling.

Different types of organic acids (aliphatic and aromatic) were used to condense with $o-$ phenylenediamine (Table 1). It has been generally observed that the presence of electron withdrawing groups in the aromatic ring enhances the reaction yield with reduced reaction time. Tri-carboxylic acid (Table 1, entry 10a) does not form any product even after sufficient time when reacted with o-phenylenediamine in 1:3 ratios. However, after $5 \mathrm{~h}$ the whole reaction mixture turns into a black solid, which is not soluble in common organic solvents. Hence, the product can be neither isolated nor characterized. The structures of the products were determined from their spectroscopic data. 
Table 1. Reaction of $o$-phenylenediamine with organic acids<smiles>[R]c1nc2ccc(CC(C)C)cc2[nH]1</smiles>

\begin{tabular}{clcc}
\hline Entry & Organic acid & Time (h) & Yield $\%^{a}$ \\
\hline 1a & Formic acid & 0.5 & 95 \\
2a & Acetic acid & 0.5 & 90 \\
3a & Benzoic acid & 1.5 & 83 \\
4a & p-Chlorobenzoic acid & 1.5 & 92 \\
5a & p-Hydroxybenzoic acid & 2 & 85 \\
6a & p-Nitrobenzoic acid & 1.5 & 95 \\
7a & p-Methoxybenzoic acid & 2 & 80 \\
8a & o-Chlorobenzoic acid & 2 & 88 \\
9a & $p$-Methylbenzoic acid & 2 & 80 \\
10a & Trimesic acid & $>2$ & No reaction \\
\hline
\end{tabular}

${ }^{a}$ Isolated yield.

Table 2. Reaction of $o$-phenylenediamine with aldehydes<smiles>[R]c1nc2ccccc2[nH]1</smiles>

\begin{tabular}{clcc}
\hline Entry & Organic aldehyde & Time (h) & ${\text { Yield } \%^{a}}^{a}$ \\
\hline 1b & Benzaldehyde & 1 & 88 \\
2b & p-Methyl benzaldehyde & 1.5 & 72 \\
3b & p-Ethyl benzaldehyde & 1.5 & 65 \\
4b & p-Nitro benzaldehyde & 0.5 & 92 \\
5b & p-Chloro benzaldehyde & 1 & 75 \\
6b & o-Chloro benzaldehyde & 1 & 70 \\
7b & p-Methoxy benzaldehyde & 1.5 & 78 \\
8b & 2-Naphthaldehyde & 2 & 61 \\
9b & 1-Hexanal & 2 & 65 \\
10b & Furan-2-carboxaldehyde & 2 & 55 \\
\hline
\end{tabular}

${ }^{a}$ Isolated yield. 
Several aldehydes (aromatic, heteroaromatic and aliphatic) underwent the above conversion to form a series of benzimidazoles (Table 2). The aldehyde group initially forms an electrophilic imine linkage by reaction with one of the amine groups of the $o$-phenylenediamine. It was followed by intra-molecular nucleophilic substitution by the remaining amine group in the orthoposition. ${ }^{13}$ Aromatic aldehydes containing both electron-donating and electron-withdrawing groups worked well. Aliphatic aldehydes (Table 2, entry 9b) also afforded the desired products in high yields. The method is suitable for the sterically hindered aldehyde, 2-naphthaldehyde (Table 2, entry $\mathbf{8 b}$ ). The reaction conditions are simple and the products were formed in moderate to high yields (55-92\%). The structures of the products were determined from their spectroscopic data.

Next, we investigated the synthesis of 2-mercaptobenzimidazole derivatives (Table 3). They were synthesized from a mixture of ammonium thiocyanate $(1 \mathrm{mmol})$ and $o$-phenylenediamine derivatives $(1 \mathrm{mmol})$ in the presence of a catalytic amount $(0.05 \mathrm{~mol} \%)$ of $\mathrm{NH}_{4} \mathrm{Cl}$ following a similar procedure. Both components were thoroughly ground with a pestle in a mortar at room temperature in an open atmosphere until the mixture turned into a melt. The mixture was then heated to $140^{\circ} \mathrm{C}$ for $1-3$ hours. The progress of the reactions was monitored by TLC. After completion, the melt was washed with cold water and the desired product was crystallized from ethanol at low temperature. Details of the substrates used, reaction times, and yields are given in Table 3.

Table 3. Synthesis of 2-mercaptobenzimidazole derivatives

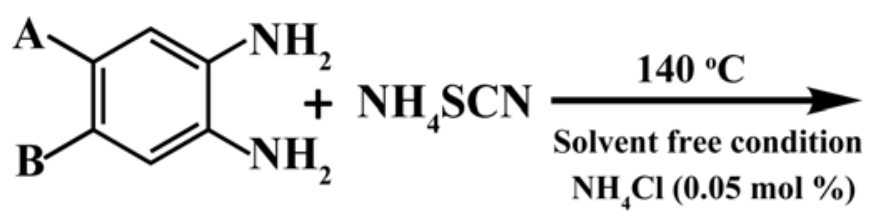<smiles>Bc1cc2[nH]c(=S)[nH]c2cc1I</smiles>

\begin{tabular}{ccccc}
\hline Entry & $\mathrm{A}$ & $\mathrm{B}$ & Time $(\mathrm{h})$ & ${\text { Yield } \%^{a}}^{a}$ \\
\hline $1 \mathrm{c}$ & $\mathrm{H}$ & $\mathrm{H}$ & 1.5 & 78 \\
$2 \mathrm{c}$ & $\mathrm{H}$ & $\mathrm{NO}_{2}$ & 3 & 48 \\
$3 \mathrm{c}$ & $\mathrm{H}$ & $\mathrm{Cl}$ & 2 & 60 \\
$4 \mathrm{c}$ & $\mathrm{H}$ & $\mathrm{Me}$ & 1 & 80 \\
$5 \mathrm{c}$ & $\mathrm{H}$ & $\mathrm{OMe}$ & 1.5 & 82 \\
$6 \mathrm{c}$ & $\mathrm{H}$ & $\mathrm{Br}$ & 1 & 85 \\
$7 \mathrm{c}$ & $\mathrm{Cl}$ & $\mathrm{Cl}$ & 3 & 54 \\
\hline
\end{tabular}

${ }^{a}$ Isolated yield. 


\section{Conclusions}

In conclusion, we have developed a simple, highly efficient, convenient, and one-pot solvent-free green synthetic method for the synthesis of biologically important benzimidazole derivatives by the condensation of $o$-phenylenediamine with either organic acid or aldehyde at $140{ }^{\circ} \mathrm{C}$. We have also described the one-pot efficient green synthetic methodology for the synthesis of another pharmaceutically important compound, 2-mercaptobenzimidazoles. Moreover, this methodology describes the synthesis without the intervention of any catalyst for the first time except for 2mercaptobenzimidazoles. It offers very attractive features such as green synthesis, reduced reaction times, and higher yields, all of which make it a useful and attractive strategy for the preparation of various benzimidazole derivatives simply by changing different substrates. The operational simplicity of the procedure is also attractive, which offers wide scope in organic synthesis.

\section{Experimental Section}

General Procedures. ${ }^{1} \mathrm{H}$ NMR and ${ }^{13} \mathrm{C}$ NMR were recorded on a Varian FT-400 $\mathrm{MHz}$ instrument. IR spectra were recorded on a Perkin-Elmer Spectrum One FT-IR spectrometer with $\mathrm{KBr}$ disks in the range $4000-400 \mathrm{~cm}^{-1}$. Elemental analyses were carried out on a PerkinElmer 2400 automatic carbon, hydrogen and nitrogen analyzer. Melting points were determined with an electrical melting point apparatus by Büchi, Switzerland. Mass spectra were recorded on a Waters Q-Tof LC-MS instrument.

This solvent-free method has an operationally simple procedure. In a typical experiment, a mixture of organic acid/organic aldehyde $(1 \mathrm{mmol})$ and $o$-phenylenediamine $(1 \mathrm{mmol})$ was thoroughly ground with a pestle in a mortar at room temperature in an open atmosphere until the overall mixture turned into a melt. The melt mixture was then heated at $140{ }^{\circ} \mathrm{C}$ for $1-2 \mathrm{~h}$. The progress of the reactions was monitored by TLC. After completion, the melt was washed with water and the desired product was crystallized from water at low temperature. The desired pure product were characterized by comparison of their physical data with those of known compounds. ${ }^{14-17}$

2-Methyl-1H-benzimidazole (2a). ${ }^{15} \mathrm{mp} 176-178{ }^{\circ} \mathrm{C},{ }^{1} \mathrm{H}$ NMR (400 MHz, $\mathrm{CDCl}_{3}$ ) $\delta: 7.20-7.43$ (m, 4H), 2.30-2.50 (m, 3H). MS, m/z: $132[\mathrm{M}]^{+}$.

2-Phenyl-1H-benzimidazole (3a). ${ }^{17} \mathrm{mp} 293-294{ }^{\circ} \mathrm{C},{ }^{1} \mathrm{H}$ NMR $\left(400 \mathrm{MHz}, \mathrm{CDCl}_{3}\right) \delta$ : $7.22(\mathrm{~m}$, 2H), 7.48 (m, 5H), 7.58 (s, $1 \mathrm{H}), 8.04$ (dd, 2H). MS, m/z: $195[\mathrm{M}+\mathrm{H}]^{+}$.

2-(4-Chlorophenyl)-1H-benzimidazole (4a). ${ }^{17} \mathrm{mp} 301-303{ }^{\circ} \mathrm{C},{ }^{1} \mathrm{H}$ NMR (400 MHz, DMSO$\left.\mathrm{d}_{6}\right): \delta 12.70(\mathrm{br} \mathrm{s}, 1 \mathrm{H}), 8.08(\mathrm{~d}, 2 \mathrm{H}), 7.59(\mathrm{~m}, 1 \mathrm{H}), 7.26-7.20(\mathrm{~m}, 3 \mathrm{H}), 7.18-7.04(\mathrm{~m}, 2 \mathrm{H}) . \mathrm{MS}$, $\mathrm{m} / \mathrm{z}: 229[\mathrm{M}+\mathrm{H}]^{+}$. 
4-(1H-Benzimidazole-2-yl)phenol (5a). ${ }^{15} \mathrm{mp} 240-242{ }^{\circ} \mathrm{C},{ }^{1} \mathrm{H}$ NMR (400 MHz, DMSO-d 6 ) $\delta$ : 8.16-8.22 (m, 2H), 7.21-7.73 (m, 6H), 4.60 (d, 1H). MS, m/z: $211[\mathrm{M}+\mathrm{H}]^{+}$.

2-(4-Nitrophenyl)-1H-benzimidazole (6a). ${ }^{15} \mathrm{mp} \mathrm{312-314}{ }^{\circ} \mathrm{C},{ }^{1} \mathrm{H}$ NMR (400 MHz, DMSO-d 6 ) $\delta: 8.00-8.08(\mathrm{~m}, 2 \mathrm{H}), 7.20-7.60(\mathrm{~m}, 6 \mathrm{H}) . \mathrm{MS}, \mathrm{m} / \mathrm{z}: 240[\mathrm{M}+\mathrm{H}]^{+}$.

2-(4-Methoxyphenyl)-1H-benzimidazole (7a). ${ }^{15} \mathrm{mp} 225-226{ }^{\circ} \mathrm{C},{ }^{1} \mathrm{H}$ NMR (400 MHz, DMSO$\left.\mathrm{d}_{6}\right) \delta: 8.00-8.08(\mathrm{~m}, 2 \mathrm{H}), 7.20-7.60(\mathrm{~m}, 6 \mathrm{H}), 3.52(\mathrm{~m}, 3 \mathrm{H}) . \mathrm{MS}, \mathrm{m} / \mathrm{z} 224[\mathrm{M}]^{+}$.

2-(2-Chlorophenyl)-1H-benzimidazole (8a). ${ }^{15} \mathrm{mp} \mathrm{232-234}{ }^{\circ} \mathrm{C},{ }^{1} \mathrm{H}$ NMR (400 MHz, DMSO-d 6 ) $\delta: ~ 8.00-8.08(\mathrm{~m}, 2 \mathrm{H}), 7.20-7.60(\mathrm{~m}, 6 \mathrm{H}) . \mathrm{MS}, \mathrm{m} / \mathrm{z}: 228[\mathrm{M}]^{+}$.

2-p-Tolyl-1H-benzimidazole (9a). ${ }^{15} \mathrm{mp} \mathrm{268-270}{ }^{\circ} \mathrm{C},{ }^{1} \mathrm{H}$ NMR (400 MHz, $\left.\mathrm{CDCl}_{3}\right) \delta: 8.00-8.08$ (m, 2H), 7.20-7.60 (m, 6H), 2.60 (m, 3H). MS, m/z: $209[\mathrm{M}+\mathrm{H}]^{+}$.

2-(4-Ethylphenyl)-1H-benzimidazole (3b). ${ }^{16} \mathrm{mp} \mathrm{285-287}{ }^{\circ} \mathrm{C},{ }^{1} \mathrm{H}$ NMR $\left(400 \mathrm{MHz}, \mathrm{CDCl}_{3}\right) \delta$ : 8.00-8.08 (m, 2H), 7.20-7.60 (m, 6H), 2.58-2.62 (m, 5H). MS, m/z: $223[\mathrm{M}+\mathrm{H}]^{+}$.

2-(Naphthalen-3-yl)-1H-benzimidazole (8b). ${ }^{16} \mathrm{mp} \mathrm{270-271}{ }^{\circ} \mathrm{C},{ }^{1} \mathrm{H}$ NMR (400 MHz, $\left.\mathrm{CDCl}_{3}\right): \delta$ 11.86 (br s, $1 \mathrm{H}), 8.74$ (br s, $1 \mathrm{H}), 8.38$ (dd, 1H), 8.02-7.90 (m, 3H), 7.69-7.52 (m, 4H), 7.26-7.18 (m, 2H). MS, m/z: $244[\mathrm{M}]^{+}$.

2-Pentyl-1H-benzimidazole (9b). ${ }^{17} \mathrm{mp} 164-165{ }^{\circ} \mathrm{C},{ }^{1} \mathrm{H}$ NMR (400 $\left.\mathrm{MHz}, \mathrm{CDCl}_{3}\right): \delta 7.21-7.43$ (m, 4H), 2.21-2.72 (m, 11H). MS, m/z: $189[\mathrm{M}+\mathrm{H}]^{+}$.

2-(Furan-2-yl)-1H-benzimidazole (10b). ${ }^{15} \mathrm{mp} \mathrm{285-287}{ }^{\circ} \mathrm{C},{ }^{1} \mathrm{H}$ NMR (400 MHz, DMSO-d 6 ):

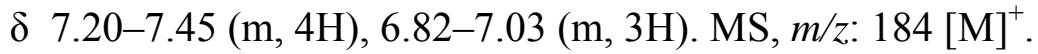

1H-Benzimidazole-2(3H)-thione (1c). ${ }^{10} \mathrm{mp} \mathrm{307-308}{ }^{\circ} \mathrm{C},{ }^{1} \mathrm{H}$ NMR (400 MHz, $\left.\mathrm{CDCl}_{3}\right): \delta 11.2$ (br s, 2H), 6.21-6.37 (m, 4H). MS, m/z: $150[\mathrm{M}]^{+}$.

5-Nitro-1H-benzimidazole-2(3H)-thione (2c). ${ }^{10} \mathrm{mp} 272{ }^{\circ} \mathrm{C},{ }^{1} \mathrm{H}$ NMR (400 MHz, DMSO-d 6 ): $\delta 12.4$ (br s, 2H), 6.47-7.30 (m, 3H). MS, m/z: $196[\mathrm{M}+\mathrm{H}]^{+}$.

5-Chloro-1H-benzimidazole-2(3H)-thione (3c). ${ }^{10} \mathrm{mp} 262{ }^{\circ} \mathrm{C},{ }^{1} \mathrm{H}$ NMR (400 MHz, DMSO-d 6 ): $\delta 11.7$ (br s, 2H), 6.15-6.38 (m, 3H). MS, m/z: $185[\mathrm{M}+\mathrm{H}]^{+}$.

5-Methyl-1H-benzimidazole-2(3H)-thione (4c). ${ }^{10} \mathrm{mp} \mathrm{290-292}{ }^{\circ} \mathrm{C},{ }^{1} \mathrm{H}$ NMR (400 MHz, DMSO-d $\left.{ }_{6}\right): \delta 12.1$ (br s, 2H), $6.01-6.54$ (m, $\left.3 \mathrm{H}\right), 2.35$ (s, 3H). MS, m/z: $165[\mathrm{M}+\mathrm{H}]^{+}$.

5-Methoxy-1H-benzimidazole-2(3H)-thione (5c). ${ }^{10} \mathrm{mp} \mathrm{255-256}{ }^{\circ} \mathrm{C},{ }^{1} \mathrm{H}$ NMR (400 MHz, DMSO-d $\left.{ }_{6}\right): \delta 11.5$ (br s, 2H), 5.72-6.10 (m, $\left.3 \mathrm{H}\right), 3.73$ (s, 3H). MS, m/z: $181[\mathrm{M}+\mathrm{H}]^{+}$.

5-Bromo-1H-benzimidazole-2(3H)-thione (6c). mp 289-292 ${ }^{\circ} \mathrm{C},{ }^{1} \mathrm{H}$ NMR (400 MHz, DMSO$\mathrm{d}_{6}$ ): $\delta 11.4$ (br s, 2H), 6.10-6.54 (m, 3H). MS, m/z; $228[\mathrm{M}]^{+}$.

5,6-Dichloro-1H-benzimidazole-2(3H)-thione (7c). ${ }^{17} \mathrm{mp} 344-346{ }^{\circ} \mathrm{C}$; ${ }^{1} \mathrm{H}$ NMR (400 MHz, DMSO-d ${ }_{6}$ ): $\delta 12.7$ (br s, 2H), $7.3(\mathrm{~d}, 2 \mathrm{H}), \mathrm{MS}: \mathrm{m} / \mathrm{z} 218[\mathrm{M}]^{+}$.

\section{Acknowledgements}

GD acknowledges DST (SR/S1/IC-01/2008) and CSIR (01(2235)/08/EMR-II) New Delhi India for financial support. We thank CIF, IIT Guwahati for NMR and Mass facility. 


\section{References}

1. (a) Gravatt, G. L.; Baguley, B. C.; Wilson, W. R.; Denny, W. A. J. Med. Chem. 1994, 37, 4338. (b) Kim, J. S.; Gatto, B.; Yu, C.; Liu, A.; Liu, L. F.; La Voie, E. J. J. Med. Chem. 1996, 39, 992. (c) Roth, T.; Morningstar, M. L.; Boyer, P. L.; Hughes, S. H.; Buckheit, R. W.; Michejda, C. J. J. Med. Chem. 1997, 40, 4199. (d) Horton, D. A.; Bourne, G. T.; Smythe, M. L. Chem. Rev. 2003, 103, 893.

2. (a) Gravatt, G. L.; Baguley, B. C.; Wilson, W. R. Denny, W. A. J. Med. Chem. 1994, 37, 4338. (b) Jayashankara, B.; Rai, K. M. L. ARKIVOC 2008, (xi), 75. (c) Roth, T.; Morningstar, M. L.; Boyer, P. L.; Hughes, S. H.; Buckheit R. W. Jr.; Michejda, C. J. J. Med. Chem. 1997, 40, 4199. (d) Lin, S. N.; Yang, L. H. Tetrahedron Lett. 2005, 46, 4315. (e) Valdez, J.; Cedillo, R.; Hernandez-Campos, A.; Yepez, L.; Hernandez-Luis, F.; NavarreteVazquez, G.; Tapia, A.; Cortes, R. Hernandezc, M.; Castilloa, R. Bioorg. Med. Chem. Lett. 2002, 12, 2221. (f) Porcari, A. R.; Devivar, R. V.; Kucera, L. S.; Drach, J. C.; Townsend, L. B. J. Med. Chem. 1998, 41, 1252. (g) Ravindra, K. C.; Vagdevi, H. M.; Vaidya, V. P. ARKIVOC 2008, (xi), 1.

3. (a) Hubschwerlen, C.; Pflieger, P.; Specklin, J. L. J. Med. Chem. 1992, 35, 1385. (b) Kim, J.

S.; Sun, Q.; Gatto, B. Bioorg. Med. Chem. 1996, 4, 621. (c) Shi, D. F., Bradshaw; T. D., Wrigley, S. J. Med. Chem. 1996, 39, 3375.

4. (a) Yun H.; Yang, J.; Baogen,W; Risen; L. Swayze, E. E. Bioorg. Med. Chem. Lett. 2004, 14, 1217. (b) Zhan, Z. H.; Liang, Y.; Yong, W. M. Catal. Commun. 2007, 8, 1126. (c) Oren, I. Y.; Yalcin, I.; Sener, E. A.; Ucarturk, N. Eur. J. Med. Chem. 2004, 39, 291. (d) Gardiner, J. M.; Colin, R.; Loyns, A. B.; Khan, A.; Mahmood, N. Bioorg. Med. Chem. Lett. 1995, 5, 1251.

5. (a) Bai, Y.; Lu, J.; Shi, Z.; Yang, B. Synlett 2001, 544. (b) Hasegawa, E.; Yoneoka, A.; Suzuki, K.; Kato, T.; Kitazume, T.; Yangi, K. Tetrahedron 1999, 55, 12957.

6. (a) Middleton, R. W.; Wibberley, D. G. J. Heterocycl. Chem. 1980, 17, 1757. (b) Hisano, T.; Ichikawa, M.; Tsumoto, K.; Tasaki, M. Chem. Pharm. Bull. 1982, 30, 2996. (c) Fairley, T. A.; Tidwell, R. R.; Donkor, I.; Naiman, N. A.; Ohemeng, K. A.; Lombardy, R. J.; Bentley, J. A.; Cory, M. J. Med. Chem. 1993, 36, 1746. (d) Czarny, A.; Wilson, W. D.; Boykin, D. W. J. Heterocycl. Chem. 1996, 33, 1393.

7. (a) Stephens, F. F.; Bower, J. D. J. Chem. Soc. 1949, 2971. (b) Chikashita, H.; Nishida, S.; Miyazaki, M.; Morita, Y.; Itoh, K. Bull. Chem. Soc. Jpn. 1987, 60, 737. (c) Kumar, S.; Kansal, V.; Bhaduri, A. Indian J. Chem. 1991, 20B, 254. (d) Patzold, F.; Zeuner, F.; Heyer, T. H.; Niclas, H. J. Synth. Commun. 1992, 22, 281. (e) Lombardy, R. L.; Tanious, F. A.; Ramachandran, K.; Tidwell, R. R.; Wilson, W. D. J. Med. Chem. 1996, 39, 1452. (f) Beaulieu, P. L.; Hache, B.; von Moos, E. Synthesis 2003, 1683.

8. (a) Zhang, Z. H.; Yin, L.; Li, Y.; Wang, Y. M. Catal. Commun. 2007, 8, 1126. (b) Zhang, Z. H.; Yin, L.; Li, Y.; Wang, Y. M. Tetrahedron Lett. 2005, 46, 889. 
9. (a) Chen, C.; Chen, Y. J. Tetrahedron Lett. 2004, 45, 113. (b) Mayer, J. P; George, S.; Lewis, C. M.; Danute, B. D. Tetrahedron Lett. 1998, 39, 6655.

10. (a) Desai, K. G.; Desai, K. R. Bioorg. Med. Chem. 2006, 14, 8271. (b) Mavrova, A. Ts.; Denkova, P.; Tsenov, Y. A.; Anichina, K. K.; Vutchev, D. I. Bioorg. Med. Chem. 2007, 15, 6291.

11. (a) Wang, M. L.; Liu, B. L. J. Chem. Eng. Jpn. 2006, 39, 633. (b) Lu, W. Z.; Liu, B.; Wei, S.; Shenzhen, D. X. Ligongban 2005, 22, 345. (c) Ling; W. M.; Liu, B. L. Chem. Eng. Commun. 1998, 165, 177.

12. (a) Thakuria, H.; Pramanik, A.; Borah, B. M.; Das, G. Tetrahedron Lett. 2006, 47, 3135. (b) Thakuria, H.; Das, G. J. Chem. Sci. 2006, 118, 425. (c) Thakuria, H.; Borah, B. M.; Das, G. J. Mol. Cat. A Chemical 2007, 274, 1.

13. Ge, F.; Wang, Z.; Wan, W.; Lu, W.; Hao, J. Tetrahedron Lett. 2007, 48, 3251.

14. Abdelkrim, B. A.; Khalid, B.; Mohamed, S. Tetrahedron Lett. 2003, 44, 5935.

15. Shen, M.; Cai, C. J. Fluorine Chem. 2007, 128, 232.

16. Das, B.; Holla, H.; Srinivas, Y. Tetrahedron Lett. 2007, 48, 61.

17. Trivedi, R., De, S. K., Gibbs, R. A. J. Mol. Cat. A Chemical 2006, 245, 8. 